\title{
Grating-based optical fiber interfaces for silicon-on-insulator photonic integrated circuits
}

\author{
G. Roelkens, D. Vermeulen, D. Van Thourhout
}

\begin{abstract}
In this paper we review our work on efficient interfaces between a silicon-on-insulator photonic integrated circuit and a single mode optical fiber based on grating structures. Several device configurations are presented that provide high-efficiency, polarization insensitive, broadband optical coupling on a small footprint. The high alignment tolerance and the fact that the optical fiber interface is out-ofplane provide opportunities for easy packaging and wafer-scale testing of the photonic integrated circuit. Finally, an optical probe based on a grating structure defined on the fiber facet is described.
\end{abstract}

Index Terms - grating couplers, silicon photonics.

\section{INTRODUCTION}

Sitong LICON Photonics is emerging as a very attractive platform o integrate optical functions on a single chip. This has several reasons. First of all, complementary metal oxide semiconductor (CMOS) fabrication tools and processes can be used to realize these devices, much in the same way as silicon electronic integrated circuits are fabricated. This welldeveloped technology infrastructure allows realizing low-cost photonic integrated circuits, given the economy of scale and the high yield of the process. In order to obtain compact photonic integrated circuits, which further reduces the cost of the integrated circuit and at the same time also reduces the power consumption and increases the electrical bandwidth of active opto-electronic devices, high refractive index contrast optical waveguide structures should be used, given the high confinement of the optical mode in such a waveguide system. Silicon-on-insulator wafers, containing a silicon waveguide layer supported by a buried $\mathrm{SiO}_{2}$ layer on top of a silicon carrier wafer provide the opportunity to realize optical waveguides with very high omni-directional refractive index contrast given the large refractive index contrast between silicon $\left(n \cong 3.45\right.$ in the near infrared) and air or $\mathrm{SiO}_{2}(n \cong 1.45)$. In order to obtain single mode operation of these silicon strip waveguide structures, the waveguide cross section is deep sub-

Manuscript received July 1, 2010. This work was supported in part by the European Integrated Project HELIOS ( $7^{\text {th }}$ framework program). G. Roelkens (e-mail: gunther.roelkens@intec.ugent.be, phone: 003292648930, fax: 003292643593), D. Vermeulen and D. Van Thourhout are with the Photonics Research Group - Ghent University/imec, Belgium. G. Roelkens acknowledges the Fund for Scientific Research Flanders (FWO) for a postdoctoral research grant. D. Vermeulen acknowledges the IWT for a $\mathrm{PhD}$ grant. micron in size, with typical dimensions of $500 \mathrm{~nm}$ in width and $200 \mathrm{~nm}$ in height. This makes efficient coupling to a standard single mode fiber far from trivial, given the large mismatch in size between the optical fiber mode and the optical mode propagating in the integrated waveguide. Even the use of endfire coupling using lensed fibers results in excessive insertion losses, when no integrated spot-size converters are used. Spotsize converters can be implemented in several ways, but the most popular approach - and the one which lends itself the most to integration in a CMOS process flow - is the use of inverted taper structures with a $\mathrm{SiON}$ or polymer overlay. While in this way high-efficiency coupling (insertion losses below $0.5 \mathrm{~dB}$ ) from a lensed optical fiber to a silicon photonic integrated circuit was demonstrated [1], this approach still lacks some features which are indispensable for low-cost packaging and testing of the photonic integrated circuit. Since packaging and testing comprises the largest fraction of the cost of a photonic IC, this is a very important issue. First of all, in order to test a particular photonic integrated circuit on an SOI wafer, the wafer needs to be diced and facets need to be polished to achieve a high-efficiency, reproducible fiber interface with the photonic circuit. This is however a very labor intensive and therefore costly operation. Moreover, lensed fibers are to be used since the topography of the spotsize converter (and hence the $\mathrm{SiON} /$ polymer overlay waveguide) needs to be kept to a minimum. 2 to $3 \mu \mathrm{m}$ thick overlay waveguides also allow realizing a semi-compact inverted taper design, with typical taper lengths on the order of $200 \mu \mathrm{m}$. This length increases exponentially when the spot size is increased. The limited converted spot size implies that still a lensed fiber is to be used and that the alignment of the lensed fiber to the facet of the photonic IC is very stringent. Active alignment can be used, but again, this is a high-cost solution. In the situation where multiple fibers in a fiber array need to interface with the IC, the coupling issues become even worse, given the relatively high variations on the fiber pitch in a fiber array and the high cost of lensed fiber arrays.

Therefore, we propose to use grating structures to interface a standard single mode optical fiber with a photonic integrated circuit. These gratings are defined on the surface of the silicon waveguide layer, as shown in figure 1. The periodic corrugation of the silicon waveguide core partially diffracts the light that impinges on the grating towards the superstrate. Therefore, positioning a standard single mode (near to) vertically on this grating coupler allows coupling light in and 
out of the photonic integrated circuit. This approach solves the

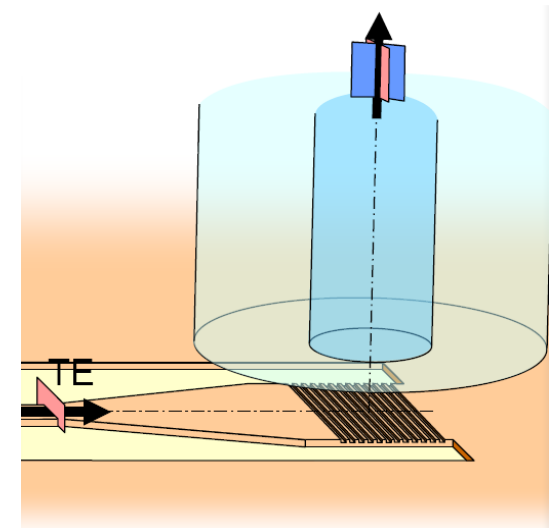

Fig. 1. Basic configuration for a fiber-to-chip grating coupler. A standard single mode fiber interfaces near vertically with the silicon photonic integrated circuit, through a diffractive grating structure defined on the surface of the silicon waveguide.

issues mentioned above: a waveguide facet is no longer required, which allows wafer-scale testing and packaging. Moreover, when the grating structure is well designed, a standard single mode fiber can be used to efficiently interface with the photonic integrated circuit, with a micrometer-level alignment tolerance. This paper deals with the design and realization of such fiber-chip interfaces. It is organized as follows: in section II we will describe the basic operation principle of this type of fiber interface. In section III we will describe means to achieve high fiber coupling efficiencies. In section IV, the optical bandwidth of fiber-chip grating couplers will be described, including multi-wavelength band operation of the devices. Section V will deal with polarization handling based on grating structures, since many photonic integrated circuits require polarization independent operation. In section VI ultra-compact implementations of these fiberinterfaces will be presented, while in section VII the interfacing to an opto-electronic component will be discussed. Finally, in section VIII we will describe an optical fiber probe based on diffractive grating structures that allows probing individual optical components in a photonic IC.

\section{GRATING COUPLER BASICS}

In its simplest form, a fiber-to-chip grating coupler consists of a periodic structure defined in a waveguide, realized by etching in a high index waveguide layer or by using deposition techniques. Light impinging on the periodic structure will diffract from the grating under an angle determined by the projected Bragg condition, which is graphically illustrated in figure 2. The $z$-component of the wave vector belonging to a certain diffraction order is given by

$$
k_{z, m}=\beta+m K
$$

in which $\beta$ is the effective propagation constant of the optical mode in the grating, $K$ is inversely proportional to the grating period $\Lambda$ and $m$ is the diffraction order. The actual diffraction angle in the superstrate and substrate is then determined by taking into account the dispersion relation in these media, for an isotropic medium given by

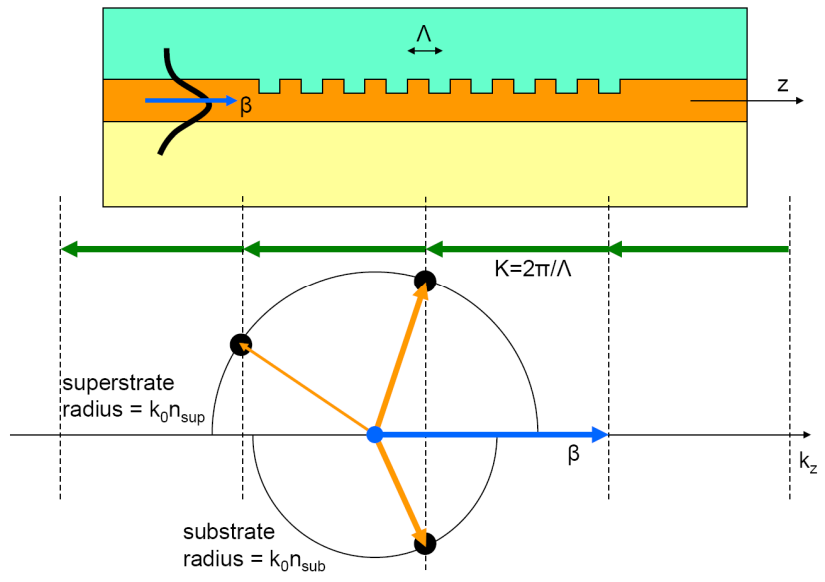

Fig. 2. Cross-section through a basic diffraction grating structure and the associated Bragg diagram, graphically illustrating the occurrence of different diffraction orders in superstrate and substrate

$$
k_{s u b, \text { sup }}=\frac{2 \pi}{\lambda} n_{\text {sub }, \text { sup }}
$$

By properly designing the grating structure, diffraction can be limited to a single diffraction order. Placing a single mode fiber under the appropriate angle with respect to the surface of the chip, therefore allows efficient coupling between the optical fiber and the photonic integrated circuit. By changing the grating period, the angle of diffraction (and hence the fiber orientation for efficient optical coupling) can be varied. At first glance, one might prefer to position the optical fiber perfectly vertical with respect to the photonic integrated circuit. This requires $K=\beta$. However, this is not a convenient choice, since this also allows another diffraction order to exist when exciting the grating from the optical waveguide, namely

$$
k_{z}=\beta-2 K=-\beta
$$

This implies that this grating structure will provide a strong reflection in the waveguide. While these reflections are as such problematic for many applications, they also result in a drastically decreased fiber-chip coupling efficiencies. By properly designing the grating, these reflections can be minimized, e.g. by etching an additional slit in from of the grating structure, which acts as an anti-reflection coating [2] or by chirping the grating [3]. However, while reflections back into the waveguide can be minimized in this way, still parasitic reflections can occur when using these structures as fiber-tochip in-couplers rather than grating out-couplers. Therefore, it is more convenient to work in a slightly "detuned" configuration in which the grating period is chosen such that the fiber is tilted slightly off-vertical (with $10^{\circ}$ off vertical being a typical number). In this way the second-order Bragg reflection is avoided, as can be seen from figure 2 .

Given the fact that the mode field diameter of a single mode optical fiber is about $10 \mu \mathrm{m}$, the diffracted field profile needs to have comparable dimensions, implying that a strong (i.e. high index contrast) grating is required. This can easily be achieved on the silicon-on-insulator waveguide platform. In 
the remainder of the paper, we will focus on this material platform. The grating coupler structures that will be discussed are designed and realized in a $220 \mathrm{~nm}$ thick silicon waveguide layer on a $2 \mu \mathrm{m}$ thick buried oxide layer, unless otherwise stated.

While the Bragg formalism discussed above allows describing the behavior of diffractive grating structures in first order, in the end rigorous simulation methods are required to fully assess the performance of a grating coupler design. For this, finite difference time domain (FDTD) methods or eigenmode expansion methods are used. For simulations, both the fiber mode (grating used as in-coupler) and the waveguide mode (grating used as out-coupler) can be excited. The modeto-mode coupling efficiency should in both cases however be the same, due to reciprocity.

\section{HIGH COUPLING EFFICIENCY GRATINGS}

A high efficiency interface between a standard single mode optical fiber and a silicon photonic integrated circuit is a prerequisite for using grating couplers in practical applications. The coupling efficiency is determined by two factors: first of all, there is the grating directionality $D$, defined as the ratio of the optical power diffracted towards the optical fiber to the total diffracted power. Secondly, the mismatch between the diffracted field profile and the Gaussian mode of the optical fiber results in additional losses. Indeed, in first order the diffracted field profile originating from a uniform grating can be considered to be exponentially decaying, with a decay length that is inversely proportional to the grating strength. This implies that there exists an optimal grating strength for which the overlap with the Gaussian fiber mode is maximal. For a fiber mode field diameter of $10.4 \mu \mathrm{m}$, this optimal field coupling strength is $0.14 / \mu \mathrm{m}$. For this optimal configuration the excess loss due to the mode profile mismatch is about $-1 \mathrm{~dB}$.

Defining a grating with this close to optimal grating strength can be done by optimizing the grating etch depth or grating fill factor (being the ratio of the etched slit width to the grating period). On a $220 \mathrm{~nm}$ silicon waveguide layer, this results in $70 \mathrm{~nm}$ deep slits (with a fill factor of $50 \%$ ) and a grating period of $630 \mathrm{~nm}$, to allow efficient coupling of $1.55 \mu \mathrm{m}$ transverse electric (TE) polarized light to a standard single mode fiber that is tilted 10 degrees off-vertical. However, rigorous numerical simulations show that the directionality of such a grating is very poor, resulting in an overall fiber-to-chip coupling efficiency of $-5 \mathrm{~dB}$ (see figure 3 ). This result implies that optimizing the grating directionality is of paramount importance to maximize the fiber coupling efficiency. In the following, we will describe several strategies to optimize the directionality.

A first strategy that can be followed is to optimize the buried oxide layer thickness. Indeed, the light that is diffracted towards the substrate first hits the $\mathrm{SiO}_{2} /$ silicon substrate interface, where a part of the light reflects back towards the grating. By properly optimizing the buried oxide layer thickness this reflection can interfere constructively with the directly upwards diffracted light, thereby leading to a larger directionaly. This however requires custom silicon-on-

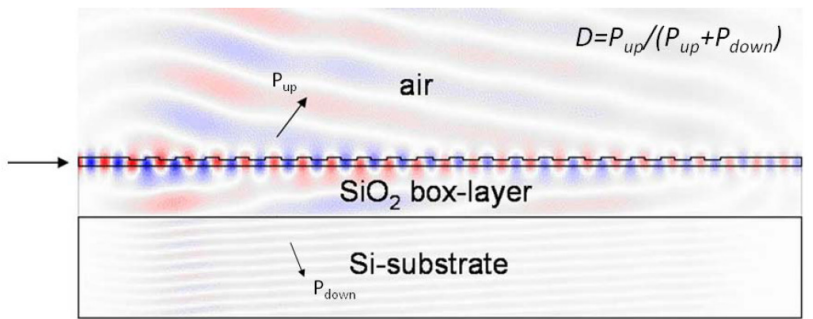

Fig. 3. Rigorous simulation of a diffraction grating defined in a $220 \mathrm{~nm}$ thick silicon waveguide layer, showing the limited directionality of this configuration
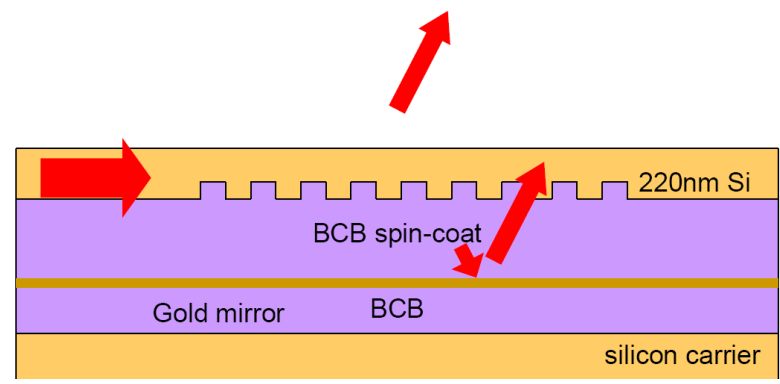

(a)

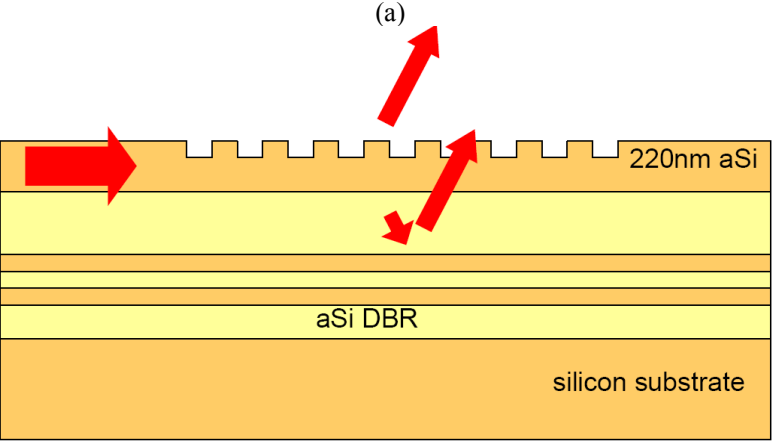

(b)

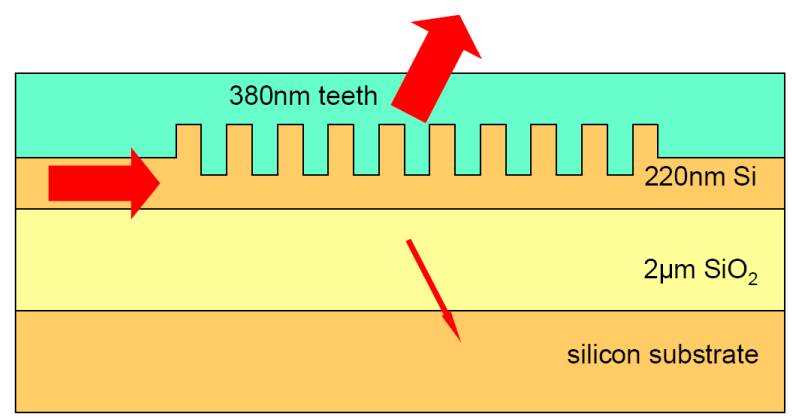

(c)

Fig. 4. Strategies to improve the grating directionality: (a) using a bottom metal mirror to redirect the downwards diffracted light, (b) using a bottom DBR mirror and (c) making the grating intrinsically directional by defining a silicon overlay.

insulator waveguide structures, making this approach less practical. Moreover, this approach only moderately improves the directionality, given the limited reflection at the $\mathrm{SiO}_{2} /$ silicon interface.

Therefore, a better approach is to apply a true bottom mirror to reflect close to $100 \%$ of the downwards diffracted light. This however still requires an optimization of the distance between the bottom reflector and the grating layer. This 
bottom mirror can be realized by means of a metallic layer or a distributed Bragg reflector (DBR), as shown in figure 4(a) and 4(b).

High efficiency grating structures for the $1550 \mathrm{~nm}$ wavelength band based were realized using a gold bottom mirror [4] and using a two-pair $\mathrm{Si} / \mathrm{SiO}_{2}$ DBR stack $[5,6]$. The gold bottom mirror based devices were realized using a DVS$\mathrm{BCB}$ wafer bonding approach. In this case the basic grating structures with limited directionality shown in figure 3 were used. On top of these diffraction gratings a DVS-BCB polymer spacer layer $(n=1.54)$ of $840 \mathrm{~nm}$ and a gold mirror of $50 \mathrm{~nm}$ were defined, after which the whole device structure was bonded up-side-down on a carrier wafer, after which the original silicon carrier wafer was removed in order to access the fiber couplers. The fabricated structures are shown in figure $5(\mathrm{a})$. In this way $-1.6 \mathrm{~dB}$ coupling efficiency was obtained for TE polarized light. While these grating structures based on metallic bottom mirrors allow for high efficiency fiber coupling, the fabrication process is not directly transferable to a CMOS line, since it requires the use of nonstandard bonding processes. Therefore a $\mathrm{Si} / \mathrm{SiO}_{2}$ DBR based approach was pursued. The fabricated device structure is shown in figure 5(b). The fiber couplers were fabricated on a $200 \mathrm{~mm} \mathrm{Si}$ wafer. First a $1 \mu \mathrm{m}$ thick $\mathrm{SiO}_{2}$ isolation layer is deposited on a bare $\mathrm{Si}$ wafer. Then the Bragg mirror, consisting of two $112 \mathrm{~nm}$ layers of amorphous silicon and a $267 \mathrm{~nm}$ layer of $\mathrm{SiO}_{2}$ are deposited. On top of the Bragg mirror a $1.48 \mu \mathrm{m}$ of buffer $\mathrm{SiO}_{2}$ and a silicon waveguide layer were deposited. Again, the basic grating structure of figure 3 was used, in order to compare the metallic mirror and DBR mirror approach. The experimentally obtained coupling efficiency is comparable to the metallic mirror approach. A combination of both techniques, i.e. using a DBR-mirror on top of crystalline silicon waveguide wafer which is then transferred to a host substrate was reported in [7].

These methods to improve the fiber coupling efficiency are quite complicated in terms of device processing. Therefore, a more elegant solution was proposed, by altering the design of the grating structure such that the grating becomes intrinsically very directional, without the need for "recycling" downwards diffracted light [8]. This approach requires the definition of a silicon overlay prior to the etching of the grating, as shown in figure 4(c).

The physical principle behind a highly directional grating as shown in Figure 4(c) can be understood as follows. Considering the diffracted field pattern as the superposition of the fields emitted by an array of scattering centers (which have a $\pi$ phase shift with respect to each other for a perfectly vertical coupling grating), constructive interference towards the superstrate (and hence the optical fiber) can be achieved by realizing an additional $\pi$ phase shift during the propagation towards the superstrate, since light is propagating either in air (in the etched slit) or in silicon (in the grating tooth). This directionality is thus a function of the waveguide thickness $h$, the etch depth $e$ in the waveguide and the silicon overlay thickness $o$. Furthermore, in order to reach high fiber coupling

efficiency, a highly directional grating is required while at the same time the grating strength needs to be optimized for maximal overlap with the Gaussian fiber mode. This modematching requirement is a function of the same three

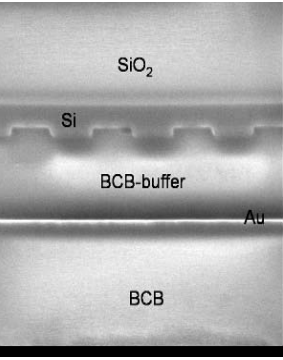

(a)

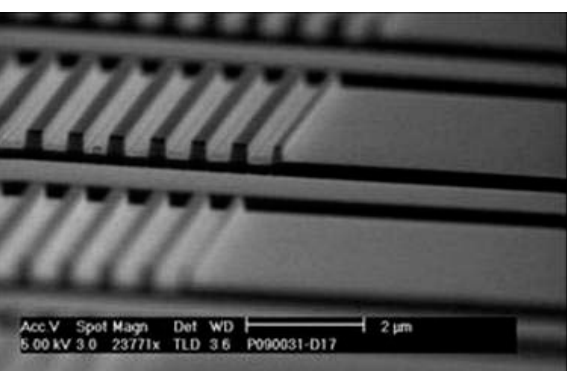

(c)

Fig. 5. Scanning electron microscope images of fabricated high-efficiency grating coupler structures based on a metal bottom mirror (a), an amorphous silicon bottom DBR (b) and a silicon overlay (c)

parameters $h, e$ and $o$ as the high directionality condition. Both conditions can be satisfied by optimizing these parameters, thereby resulting in high-efficiency coupling. The fabricated structures are shown in figure 5(c). Experimentally, again $1.6 \mathrm{~dB}$ fiber-to-chip coupling efficiency is obtained for $\mathrm{TE}$ polarized light. This grating structure is intrinsically directional, irrespective of the buried oxide layer thickness [9].

In order to further improve the fiber-chip coupling efficiency, the mismatch between the diffracted field profile and the Gaussian mode profile of the single mode fiber needs to be reduced. This can be achieved using non-uniform grating structures to gradually change the coupling strength of the grating. This way, in principle $<0.5 \mathrm{~dB}$ fiber coupling efficiency can be obtained when defining a non-uniform grating on any of the platforms described above $[10,11]$. Typically, very narrow slits (on the order of tens of nanometers) are required to achieve this goal. This is very challenging for the deep UV lithography tools that are used to define these silicon photonic waveguide circuits on 8inch SOI wafers. Therefore, we will not consider non-uniform gratings further.

The obtained coupling efficiencies mentioned require an optimization of the fiber position on top of the grating. Alignment tolerances are not very strict however, since one is aligning a single mode fiber to a spot of about $10 \mu \mathrm{m}$ in diameter. Experimentally, a $-0.5 \mathrm{~dB} \quad(-1 \mathrm{~dB})$ excess loss alignment tolerance of $+/-1.5 \mu \mathrm{m}(+/-2.5 \mu \mathrm{m})$ is obtained. This makes diffractive grating couplers a very attractive solution for the passive alignment of a single fiber or fiber array to a 
silicon photonic integrated circuit. Coupling of a fiber array to a silicon waveguide circuit was reported in $[12,13]$.

\section{GRATING COUPLER BANDWIDTH}

Since the diffraction properties of the grating structure are used to couple light between an optical fiber and the silicon waveguide circuit, the coupling efficiency is inherently wavelength dependent. However, since the considered gratings have a very short coupling length (in order to match the diffracted field size with that of the fiber mode), considerable optical bandwidth can still be obtained. This can be explained by considering the exponentially decaying diffracted field profile as a superposition of plane waves. The angular distribution of these plane waves will become broader when the grating is shorter (stronger). This implies that a change in wavelength (implying a rotation of this angular distribution of plane waves) will have a lower impact when the grating is shorter, resulting in a larger optical bandwidth for stronger gratings. The exact optical bandwidth depends on the specific design. For the considered high efficiency grating couplers described in the previous section based on the silicon overlay grating, the $-1 \mathrm{~dB}(-3 \mathrm{~dB})$ excess loss is $45 \mathrm{~nm}(90 \mathrm{~nm})$ around a wavelength of $1.55 \mu \mathrm{m}$.

While this optical bandwidth is sufficient for many applications, there are applications which require a larger optical coupling bandwidth, especially because of the fact that light in two distinct (and widely separated) wavelength bands needs to be processed by the photonic integrated circuit. An important application satisfying this description is the use of integrated transceivers for fiber-to-the-home (FTTH) optical networks, in which at the subscriber side a $1310 \mathrm{~nm}$ wavelength is used to transmit upstream data over the network, while a $1490 \mathrm{~nm}$ downstream optical signal needs to be processed (and vice versa at the central office side). As the wavelength span required in this application exceeds the optical bandwidth of the fiber-to-waveguide grating coupler, an alternative approach is needed, which increases the effective wavelength span, while maintaining the advantage of being compact and allowing wafer-scale testing and ease of packaging.

The concept of fiber-chip coupling using diffraction gratings can however be extended to allow two different wavelength bands to interface with the photonic integrated circuit. This is illustrated in figure 6 , showing essentially the same type of grating structures as discussed above, however now considering the two access waveguides to the one-dimensional grating (left and right access waveguide) [14]. By optimizing the grating geometry and the fiber tilt angle, two distinct wavelength bands can be coupled into and out of the waveguide circuit, e.g. the $1310 \mathrm{~nm}$ wavelength band and the $1490 \mathrm{~nm}$ wavelength band. The maximum fiber-to-chip coupling efficiency that can be obtained in this way is lower than for the case of a single wavelength band, since there is a trade-off between both wavelength bands. As shown in figure $6(\mathrm{~b})$, the coupling efficiency reaches about $-2.5 \mathrm{~dB}$ for both the $1310 \mathrm{~nm}$ and $1490 \mathrm{~nm}$ wavelength band, with a $-3 \mathrm{~dB}$ excess loss bandwidth of $55 \mathrm{~nm}$ to $60 \mathrm{~nm}$ for the silicon overlay design. These grating structures couple two wavelength bands to the silicon waveguide circuit, while at the same time spatially separating them on the chip. Therefore, this type of grating is also referred to as a grating duplexer.

These duplexing grating structures, as well as the

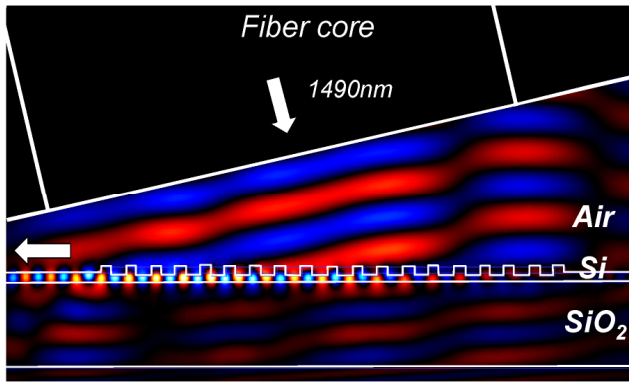

(a)

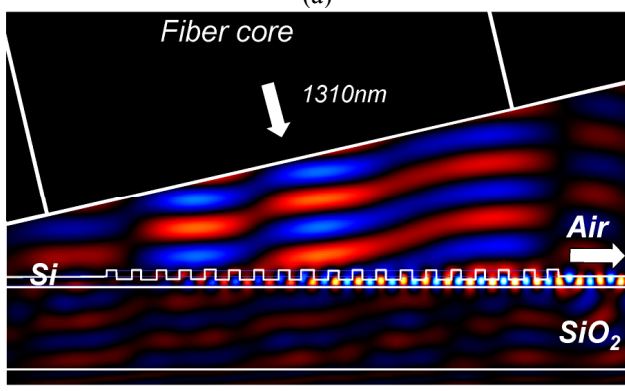

(b)

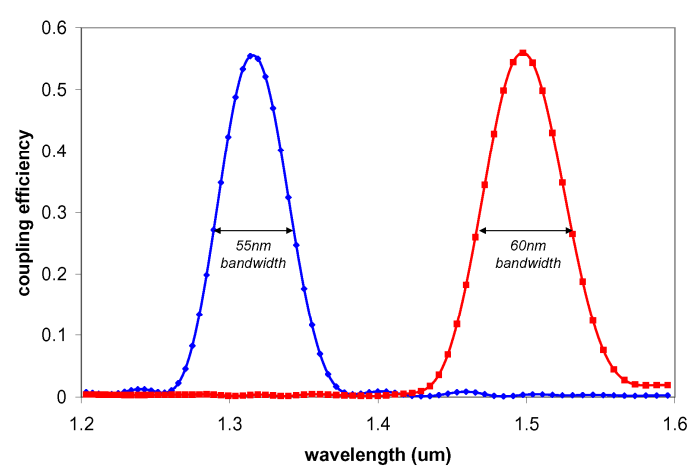

(c)

Fig. 6. One-dimensional grating duplexer design operating in the $1.3 \mu \mathrm{m}$ and $1.49 \mu \mathrm{m}$ wavelength range

one-dimensional grating structures described in section III, perform very differently for TE and TM polarized light. This is an intrinsic problem for high index contrast waveguide structures. In many practical applications (such as the FTTH application mentioned above) polarization independent operation is required however. This will be discussed in the following section.

\section{POLARIZATION HANDLING}

All high index contrast, high confinement waveguide structures are intrinsically very polarization dependent. This implies that realizing an intrinsically polarization independent photonic integrated circuit on the silicon-on-insulator platform discussed in this paper is nearly impossible. However, the problem of polarization handling can be solved by using a 
polarization diversity configuration. In this configuration, the two orthogonal polarizations in the optical fiber are both coupled to the photonic integrated circuit, but into a different waveguide. Thereby, the two polarizations are split on the chip and can be further processed by two parallel photonic integrated circuits. This implies that polarization independent operation can be achieved, at the expense of doubling the size of the integrated circuit. Polarization diversity can be implemented very elegantly using diffraction grating structures. Both one-dimensional and two-dimensional diffraction gratings can be used for this purpose.

The operation principle of a two-dimensonal grating structure is outlined in figure 7, showing how a square lattice of holes (that can be considered as the superposition of two orthogonal one-dimensional grating structures) efficiently couples both orthogonal polarizations to the chip. The coupling is such that the orthogonal polarizations in the optical fiber are coupled to essentially identical polarizations (in our case TE) in two orthogonal waveguides. This two-dimensional grating structure therefore at the same time performs the function of a fiber-chip interface, a polarization splitter and polarization rotator for a polarization diversity approach, all on a $10 \mu \mathrm{m}$ by $10 \mu \mathrm{m}$ footprint.

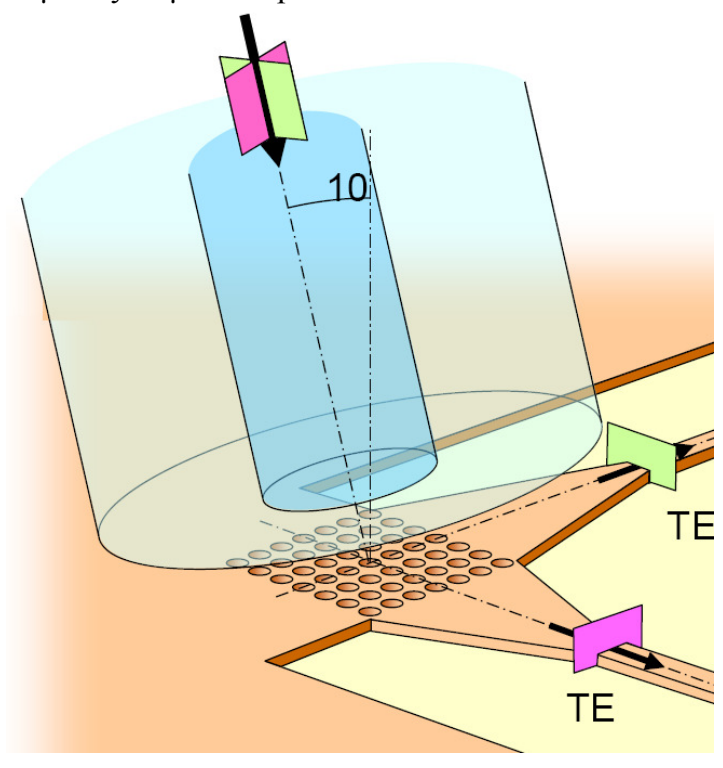

Fig. 7. Two-dimensional grating coupler for polarization handling on a silicon photonic integrated circuit

Experimental realizations of these two-dimensional structures, by etching a square lattice of holes $70 \mathrm{~nm}$ deep into the silicon waveguide layer show intrinsically the same efficiency as the standard one-dimensional grating structures as shown in figure 3. Quasi-polarization independent operation was obtained in a $10 \mathrm{~nm}$ wavelength range around the grating coupler coupling efficiency maximum (below $1 \mathrm{~dB}$ polarization dependent loss) [15]. Outside this wavelength range, the polarization dependent loss becomes too high for practical purposes. The origin of the residual polarization dependent loss and the strong wavelength dependent behavior is the tilting of the optical fiber. In case the fiber is positioned perfectly vertically on top of the $2 \mathrm{D}$ grating structure, the polarization dependent loss is intrinsically zero (provided that the optical fiber is well aligned on the grating structure). As discussed in section II, the perfectly vertical orientation of the optical fiber is however not convenient due to the presence of second order Bragg reflection. Therefore the fiber is tilted off-vertical, along the bisection line of the 2D grating structure as shown in figure 7 . This configuration is schematically outlined in figure 8, illustrating the use of $2 \mathrm{D}$ gratings in a polarization diversity scheme with an off-vertical

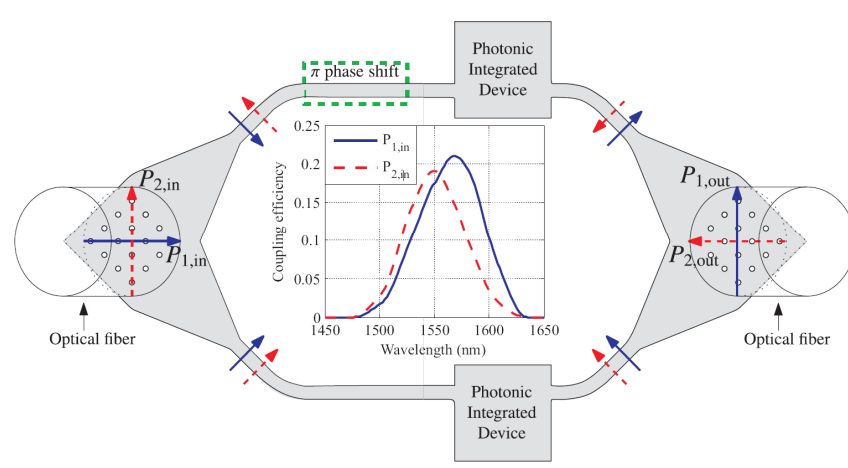

Fig. 8. Polarization diversity waveguide circuit with an introduced $\pi$ phase shift to eliminate residual polarization dependent loss originating from the tilting of the optical fibers

fiber interface [16]. Two orthogonal polarizations in the optical fiber, $\mathrm{P}_{1}$ and $\mathrm{P}_{2}$, where $\mathrm{P}_{1}$ lies along the bisection line of the photonic integrated circuit are to be considered. In case the optical fiber is perfectly vertical, both polarizations experience the same fiber coupling efficiency. Polarization $\mathrm{P}_{1}$ equally excites the TE polarized waveguide mode in the upper and lower waveguide arm and both are excited in phase. For polarization $\mathrm{P}_{2}$ the TE polarized waveguide modes in both waveguide arms are also excited equally, however this time with a $\pi$ phase shift between both arms of the polarization diversity circuit. When the optical fiber in now tilted offvertical, along the bisection line of the polarization diversity circuit, Polarization $\mathrm{P}_{1}$ and $\mathrm{P}_{2}$ will no longer experience the same fiber-chip coupling efficiency, since polarization $P_{1}$ is tilted out of plane. This is shown in the inset of figure 8 (result of 3D FDTD simulations). This tilting of the optical fiber therefore leads to an intrinsic polarization dependent loss of the $2 \mathrm{D}$ grating coupler. An elegant way to circumvent this PDL for fiber to fiber connected photonic integrated circuits is to introduce an additional $\pi$ phase shift between both arms of the photonic IC. Indeed, adding this $\pi$ phase shift renders the TE modes excited by polarization $\mathrm{P}_{2}$, which were initially out of phase, in phase at the 2D grating out-coupler. This implies that these modes will recombine in the output fiber as polarization $\mathrm{P}_{1}$, and vice versa for the other polarization state. The implication of this $\pi$ phase shift is therefore that, when looking at the fiber-to-fiber transmission spectrum, both polarizations experience once the fiber-chip coupling curve for polarization $\mathrm{P}_{1}$ and $\mathrm{P}_{2}$, thereby eliminating the polarization dependent loss originating from the tilt of the optical fiber. This was experimentally verified by applying a heater on top of one arm of the polarization diversity circuit as shown in figure 8 , to introduce a phase shift between both arms. At the same time the polarization dependent loss of the circuit (which 
was in this case a simple ring resonator waveguide circuit) was monitored. Low polarization dependent loss in this type of polarization diversity circuit however also relies on the fact that the actual photonic integrated circuit in both arms of the polarization diversity configuration needs to be identical. This is far from trivial in high index contrast waveguide circuits, even using state-of-the-art fabrication technologies. For example, if the waveguide width of a silicon-on-insulator ring resonator varies by $1 \mathrm{~nm}$, also the resonance wavelength of the resonator shifts by approximately $1 \mathrm{~nm}$. This implies that we typically will

also

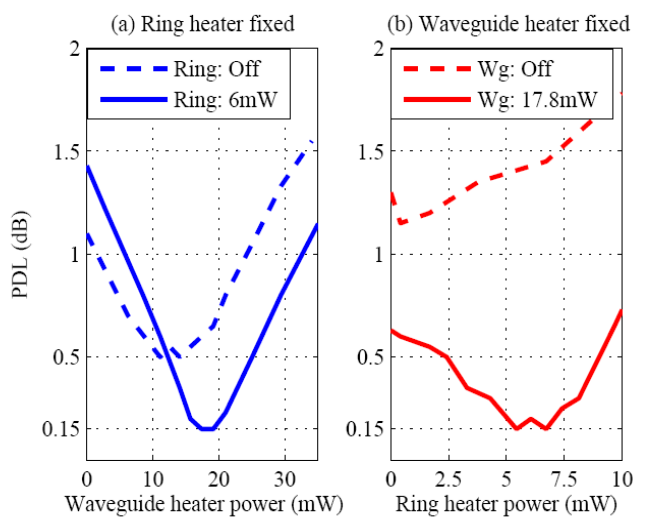

(a)
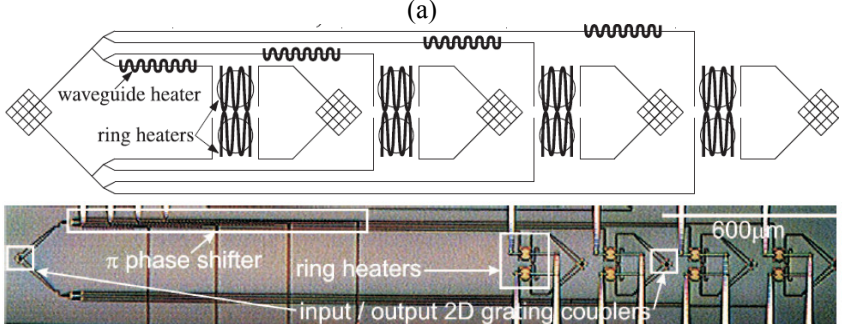

(b)

Fig. 9. Influence of the waveguide heater power and ring resonator heater power on the residual PDL of the polarization diversity waveguide circuit (a) shown in (b).

need to be able to trim both waveguide circuits to make them truly identical. In some circuit configurations this problem can be circumvented by using a single photonic integrated circuit in which light propagates in opposite directions [17]. In figure 9 we plot the residual polarization dependent loss for a varying waveguide heater power (i.e. phase shift) and for a varying ring resonator heater power (used to trim the ring resonator to make both arms of the polarization diversity circuit identical). Indeed, a large reduction in polarization dependent loss can be observed, from $1.5 \mathrm{~dB}$ without any heater control, down to $0.15 \mathrm{~dB}$ in the optimal configuration. Low PDL values (i.e. below $1 \mathrm{~dB}$ ) for a given waveguide heater and ring heater power dissipation can also be obtained over a larger wavelength range (i.e. $25 \mathrm{~nm}$ ).

A second implementation of a grating structure for a polarization diversity configuration is based on a onedimensional line grating structure. Line grating structures have the advantage that they can be optimized more easily than square lattice grating structures (given the quasi twodimensional behavior of the line gratings) and hence ultimate performance can be expected from these devices. The configuration that is considered is shown in figure 10. Again, both access waveguides to the one-dimensional grating structure are used and a silicon overlay is used to boost the efficiency of the grating structure. The operation principle is comparable to the grating duplexer described in section IV, except now the TE and TM polarizations at the same wavelength are considered (compared to the TE polarized modes at two different wavelengths for the grating duplexer) $[18,19]$. Proper design of the grating allows for quasi-identical fiber-to-chip coupling efficiencies for both polarizations over a limited wavelength range, hence resulting in a low polarization dependent loss of the 1D grating structure, as shown in figure $10(\mathrm{~b})$, in this case for the $1310 \mathrm{~nm}$

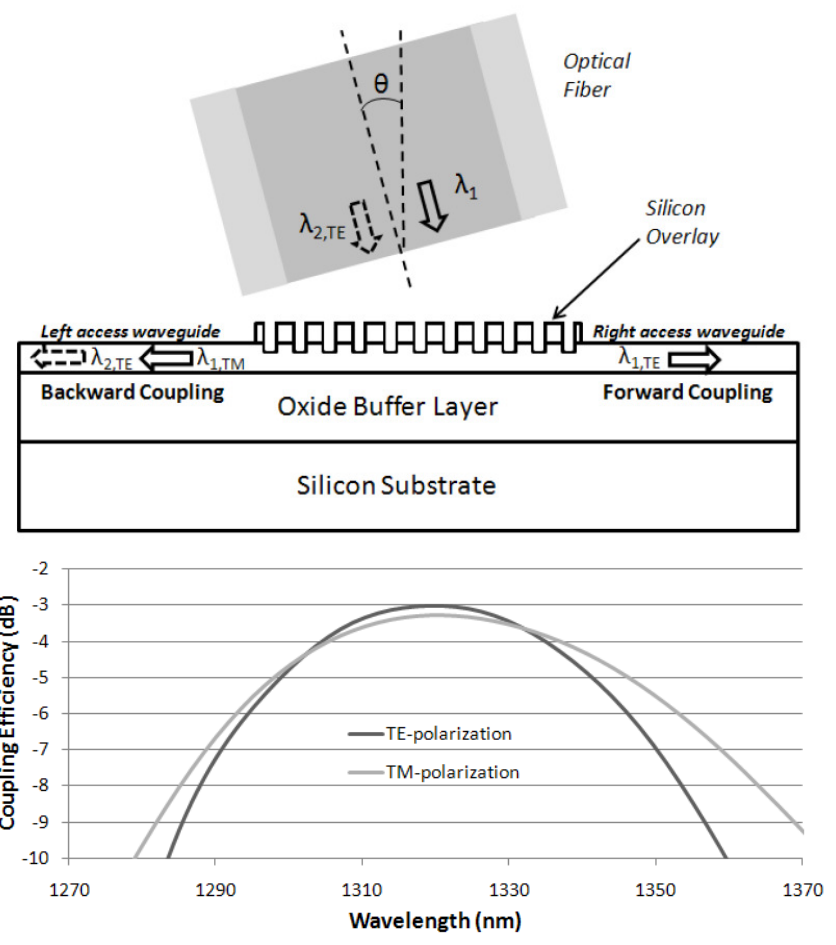

Fig. 10. One-dimensional grating configuration for a polarization diversity waveguide circuit, allowing polarization independent handling of a single wavelength band $\left(\lambda_{1}\right)$, while also accommodating coupling of a second wavelength band $\left(\lambda_{2}\right)$ for a single polarization

wavelength band. Also experimentally a low PDL was obtained, i.e. from $1240 \mathrm{~nm}$ to $1310 \mathrm{~nm}$ the fiber-to-fiber PDL is lower than $1 \mathrm{~dB}$, which covers $42 \mathrm{~nm}$ within the $50 \mathrm{~nm}-3 \mathrm{~dB}$ coupling efficiency bandwidth of the grating coupler [19]. Since the device configuration is identical to the grating duplexer configuration described in section IV, an additional wavelength band $\lambda_{2}$ can be accommodated, although only for a single polarization. In order to build true polarization diversity circuits based on these one-dimensional grating couplers, an additional polarization rotator is required to obtain identical polarizations in both arms of the circuit. Several implementations of a polarization rotator on the silicon waveguide platform can be considered [20,21]. 


\section{ULTRA-COMPACT IMPLEMENTATIONS}

The diffraction grating structures considered in this paper are very compact, since they only occupy the area of the core of a standard single mode optical fiber, roughly $10 \mu \mathrm{m}$ by $10 \mu \mathrm{m}$. However, in order to convert the $10 \mu \mathrm{m}$ wide optical waveguide mode to that of a $0.5 \mu \mathrm{m}$ single mode waveguide, a relatively long adiabatic taper is required, on the order of $150 \mu \mathrm{m}$ in length. This makes the total device structure less compact, thereby increasing the overall footprint of the photonic integrated circuit. One elegant way to circumvent this issue is to use focusing grating structures, instead of the waveguide structures described above. A one-dimensional grating structure can be made to focus the coupled beam on a micrometer size aperture by curving the grating lines, as shown in figure $11[22,23]$. In this case, each grating line represents a part of an ellipse, while

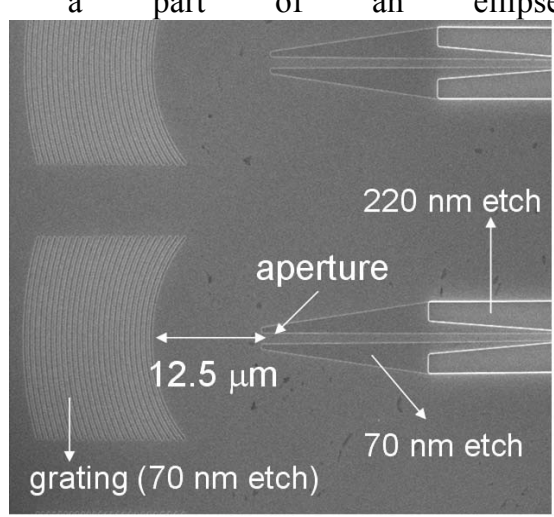

(a)

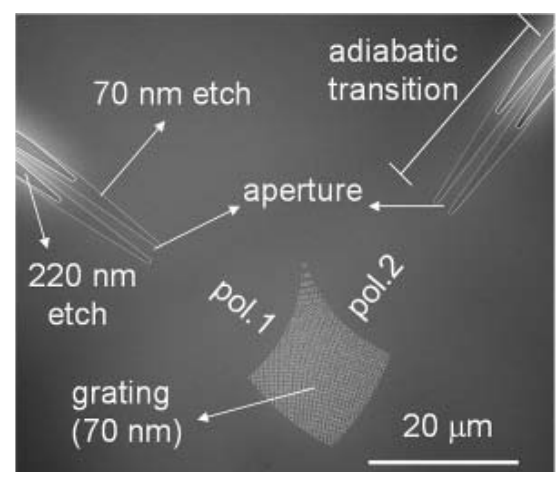

(b)

Fig. 11. Influence of the waveguide heater power and ring resonator heater power on the residual PDL of the polarization diversity waveguide circuit (a) shown in (b).

all ellipses describing the focusing grating have a common focal point, namely at the waveguide aperture. A similar design consideration can be made for two-dimensional grating couplers, by considering them as a "superposition" of two onedimensional curved grating structures. This way a twodimensional grating structure can be designed by implementing a scattering center (i.e. an etched hole) at the intersection of the two orthogonal curved one-dimensional gratings. This approach can dramatically decrease the overall footprint of the device, as shown in figure 11 (in this case for the standard grating configuration of figure 3), without impacting the overall performance of the device in terms of fiber coupling efficiency, alignment tolerance and polarization dependent loss.

\section{INTERFACING TO OPTO-ELECTRONIC COMPONENTS}

So far, the grating structures were considered as an efficient interface between a silicon photonic integrated circuit and a single mode optical fiber. However, its use can be extended to also interface with opto-electronic components (lasers, photodetectors,...) integrated on the silicon platform. It is easy to envision the integration of a III-V semiconductor photodetector on top of a grating coupler, either through flipchip integration or heterogeneous integration. An example of such a heterogeneously integrated III-V/silicon device is shown in figure $12[24,25]$. In this case a III-V epitaxial layer stack

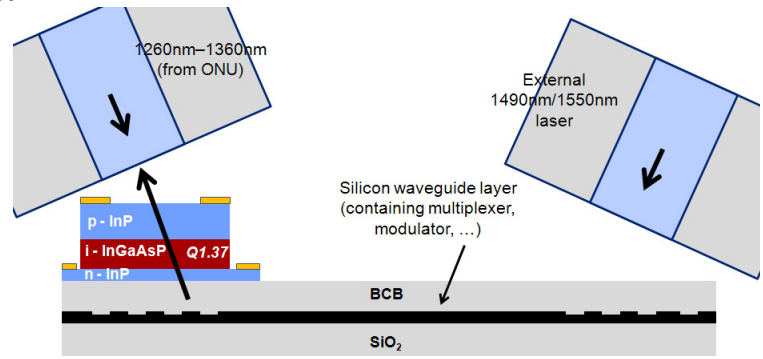

(a)

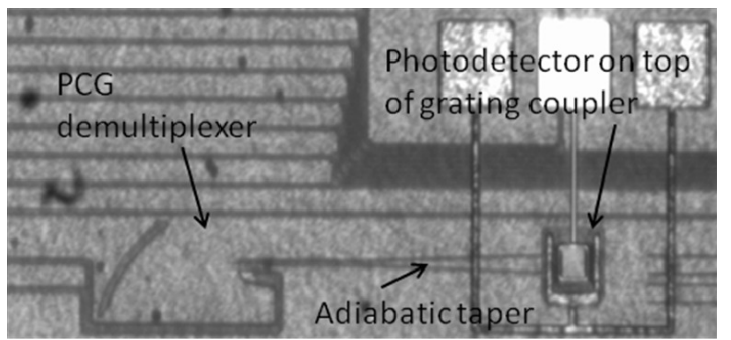

(b)

Fig. 12. Example of the integration of a photodetector on top of a diffractive grating coupler

transferred to a silicon waveguide circuit by means of a BCB adhesive bonding process [26]. This silicon waveguide circuit contains diffraction gratings for vertical interfacing with both an optical fiber and the photodetectors defined in the III-V epitaxial layer.

Besides interfacing with photodetectors, also the coupling of laser emission into the photonic integrated circuit can be envisioned. This is however complicated by the fact that the laser beam needs to be coupled in near-vertically (but not perfectly vertical) in order to be efficiently coupled to the waveguide circuit. This requires either micro-optics (reflecting mirrors or refractive wedges) or a tilted mounting of the laser.

\section{OPTICAL FIBER PROBE}

One very attractive feature of grating couplers is that they allow interfacing with a photonic integrated circuit on a wafer scale. This allows for wafer-scale testing and packaging of the photonic integrated circuit. However one would often want to 
be able to probe individual optical components of a complex interconnected silicon photonic integrated circuit, e.g. for debugging purposes. This can be achieved using an optical fiber probe, in which the diffractive grating structure is defined on the facet of a single mode optical fiber [27].

In figure 13(a) the method to define this grating structure is outlined. A gold grating was defined on a fiber surface using a nano-imprint technique. First, the fiber with UV-curable resist is aligned over the specially prepared mold carrying the 10 by $10 \mu \mathrm{m}$ gold grating pattern in the trenches of the mold. Upon attachment, the cavities are filled and the resist is UV cured. Finally, the mold is released. The metal grating is now attached to the fiber, due to the fact that the mold was covered with an anti-stiction film prior to gold deposition. By using gold as the grating material, a high refractive index contrast is obtained. A fabricated optical probe is shown in figure 13(b). This way efficient coupling between the optical probe and the photonic integrated circuit can be obtained. Experimentally
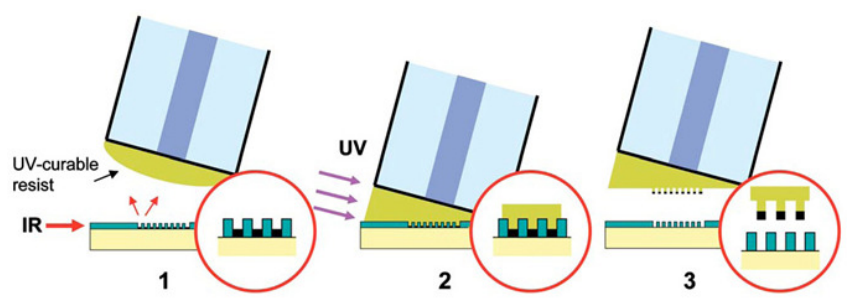

(a)
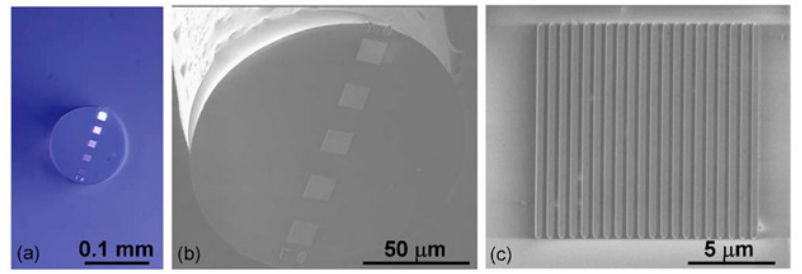

(b)

Fig. 13. Fabrication procedure to define a gold grating on a single mode fiber facet

$15 \%$ coupling efficiency between a single mode fiber probe and a $3 \mu \mathrm{m}$ wide photonic integrated circuit was realized for TE polarized light at $1.55 \mu \mathrm{m}$ wavelength. The grating consisted of $20 \mathrm{~nm}$ thick gold stripes on a $630 \mathrm{~nm}$ pitch. The realization of this optical probe can pave the way to waferscale testing of high index contrast large scale integrated waveguide circuits.

\section{CONCLUSIONS}

Photonic integrated circuits have tremendous benefits over their discrete counterparts. Especially the use of high index contrast waveguide structures for photonic integrated circuits allows large scale integration of optical functions on a single substrate, reducing the cost, size and weight of the devices, while improving their performance. Silicon photonics is emerging as a very promising platform for photonic integration, since the economy of scale in fabrication will make the photonic integrated circuits virtually free. The cost of the packaging and testing of these integrated circuits is therefore the predominant cost and performance issue and is one of the most important aspects for bringing high index contrast waveguide structures from research to practical applications. In this paper we show that the use of diffractive grating structures could provide a low-cost solution for this problem, since wafer-level testing and packaging can be realized without the need for polished facets.

\section{ACKNOWLEDGEMENT}

The authors would like to acknowledge the many people that were involved in the design, fabrication and testing of various types of grating couplers, including D. Taillaert, S. Selveraja, F. Van Laere, W. Bogaerts, S. Scheerlinck, P. Dumon, J. Brouckaert, J. Schrauwen, P. Verheyen and G. Lepage.

\section{REFERENCES}

[1] S. McNab, N. Moll, and Y. Vlasov, "Ultra-low loss photonic integrated circuit with membrane-type photonic crystal waveguides," Optics Express, vol. 11, no. 22, pp. 2927-2939, 2003.

[2] G. Roelkens, D. Van Thourhout, and R. Baets, "High Efficiency grating coupler between silicon-on-insulator waveguides and perfectly vertical optical fibers," Opt. Lett. 32, 1495-1497 (2007).

[3] X. Chen, C. Li, and H. K. Tsang, "Fabrication-tolerant waveguide chirped grating coupler for coupling to a perfectly vertical optical fiber," IEEE Photonics Technology Letters, 20, 1914-1916 (2008).

[4] F. Van Laere, G. Roelkens, M. Ayre, J. Schrauwen, D. Taillaert, D. Van Thourhout, T. F. Krauss, and R. Baets, "Compact and highly efficient grating couplers between optical fiber and nanophotonic waveguides," J. Lightwave Technology, 25, 151-156 (2007).

[5] S. K. Selvaraja, D. Vermeulen, M. Schaekers, E. Sleeckx, W. Bogaerts, G. Roelkens, P. Dumon, D. Van Thourhout, and R. Baets, "Highly efficient grating coupler between optical fiber and silicon photonic circuit," in Lasers and Electro-Optics/International Quantum Electronics Conference, (2009).

[6] KTH paper

[7] HELIOS paper

[8] G. Roelkens, D. Van Thourhout, and R. Baets, "High efficiency siliconon-insulator grating coupler based on a poly-silicon overlay," Opt. Express 14, 11622-11630 (2006)

[9] D. Vermeulen, S. Selvaraja, P. Verheyen, G. Lepage, W. Bogaerts, G. Roelkens, High-efficiency Silicon-On-Insulator Fiber-to-Chip Grating Couplers Using a Silicon Overlay, Group IV photonics, United States, p.FPd1 (2009)

[10] D. Taillaert, P. Bienstman, and R. Baets, "Compact efficient broadband grating coupler for silicon-on-insulator waveguides," Opt. Lett. 29, 2749-2751 (2004).

[11] Y. Tang, Z. Wang, L. Wosinski, U. Westergren, and S. He, "Highly efficient nonuniform grating coupler for silicon-on-insulator nanophotonic circuits," Opt. Lett., vol. 35, pp. 1290-1292 (2010).

[12] P. Dumon, W. Bogaerts, D. Van Thourhout, D. Taillaert, R. Baets, J. Wouters, S. Beckx, P. Jaenen, Compact wavelength router based on a Silicon-on-insulator arrayed waveguide grating pigtailed to a fiber array, Optics Express, 14(2), p.664-669 (2006)

[13] L. Zimmermann, T. Tekin, H. Schroeder, P. Dumon, and W. Bogaerts, How to bring nanophotonics to application - silicon photonics packaging, IEEE LEOS Newsletter, 2008

[14] G. Roelkens, D. Van Thourhout, R. Baets, silicon-on-insulator ultacompact duplexer based on a diffractive grating structure, Optics Express, 15(16), p.10091 (2007)

[15] G. Roelkens, D. Vermeulen, F. Van Laere, S. Selvaraja, S. Scheerlinck, D. Taillaert, W. Bogaerts, P. Dumon, D. Van Thourhout, R. Baets, Bridging the Gap Between Nanophotonic Waveguide Circuits and Single Mode Optical Fibers Using Diffractive Grating Structures , Journal of Nanoscience and Nanotechnology, 10, p.1551-1562 (2010)

[16] R. Halir, D. Vermeulen, G. Roelkens, Reducing polarization dependent loss of silicon-on-insulator fiber to chip grating couplers, IEEE Photonics Technology Letters, 22(6), p.389-391 (2010) 
[17] W. Bogaerts, D. Taillaert, P. Dumon, D. Van Thourhout, R. Baets, A polarization-diversity wavelength duplexer circuit in silicon-on-insulator photonic wires, Optics Express, 15(4), p.1567-1578 (2007)

[18] D. Vermeulen, G. Roelkens, D. van Thourhout, Silicon-on-Insulator Dual-Band Fiber-To-Chip 1D Grating Couplers for Fiber-To-The-Home (FTTH) Integrated Transceivers, submitted to IEEE Photonics Journal

[19] Z. Wang, Y. Tang, N. Zhu, L. Wosinski, D. Dai, U. Westergren, and S. He, "Experimental Demonstration of an Ultracompact Polarization Beam Splitter Based on a Bidirectional Grating Coupler," OSA Asia Communications and Photonics Conference and Exhibition (ACP), 2009, November 2-6, 2009, Shanghai, China.

[20] D. Vermeulen, S. Selvaraja, W. Bogaerts, G. Roelkens, High-efficiency broadband CMOS-compatible polarization rotator on SOI,accepted for publication in Group IV photonics, China, (to be published)

[21] Fukuda H, Yamada K, Tsuchizawa T, Watanabe T, Shinojima H, and Itabashi S., Polarization rotator based on silicon wire waveguides, Opt Express. 2008 Feb 18;16(4):2628-35.

[22] F. Van Laere, T. Claes, J. Schrauwen, S. Scheerlinck, W. Bogaerts, D. Taillaert, L. O'Faolain, D. Van Thourhout, R. Baets, Compact Focusing Grating Couplers for Silicon-on-Insulator Integrated Circuits,Photonics Technology Letters, 19(23), p.1919-1921 (2007)

[23] F. Van Laere, W. Bogaerts, P. Dumon, G. Roelkens, D. Van Thourhout, R. Baets, Focusing polarization diversity grating couplers in silicon-oninsulator,Journal of Lightwave Technology, 27(5), p.612-618 (2009)

[24] G. Roelkens, J. Brouckaert, D. Taillaert, P. Dumon, W. Bogaerts, D. Van Thourhout, R. Baets, Integration of InP/InGaAsP photodetectors onto Silicon-on-Insulator waveguide circuits, Optics Express, 13(25), p.10102-10108 (2005)

[25] D. Vermeulen, T. Spuesens, P. De Heyn, P. Mechet, R. Notzel, S. Verstuyft, D. Van Thourhout, G. Roelkens, III-V/SOI photonic integrated circuit for FTTH central office transceivers in a PTP network configuration, accepted for publication in ECOC, Italy

[26] G. Roelkens, J. Brouckaert, D. Van Thourhout, R. Baets, R. Notzel, M. Smit, Adhesive Bonding of InP/InGaAsP Dies to Processed Silicon-onInsulator Wafers using DVS-bis-Benzocyclobutene,Journal of Electrochemical Society, 153(12), p.G1015-G1019 (2006)

[27] S. Scheerlinck, D. Taillaert, D. Van Thourhout, R. Baets, Flexible metal grating based optical fiber probe for photonic integrated circuits, Applied Physics Letters, 92(3), p.031104 (2008)

[28] G. O. Young, "Synthetic structure of industrial plastics (Book style with paper title and editor)," in Plastics, 2nd ed. vol. 3, J. Peters, Ed. New York: McGraw-Hill, 1964, pp. 15-64.

G. Roelkens graduated in 2002 as an electronics engineer (option: microelectronics and opto-electronics) from Ghent University (with highest honour). Since 2002, he has been working in the Photonics Research Group of prof. Roel Baets at Ghent University, where he received the doctoral degree in April 2007, for his work in the field of heterogeneous III-V/Silicon photonics. In this work, the technology for integrating III-V material on top of silicon-on-insulator waveguide circuits was developed and the integration of thin film III-V laser diodes and photodetectors on top of and coupled to the SOI waveguide circuit was demonstrated. Now he is working as a post-doc in the same group (on a three year post-doctoral research grant). His research interests include heterogeneous III-V/silicon integration, efficient fiber-chip coupling, all-optical signal processing and mid-infrared photonic integrated circuits.

D. Vermeulen graduated in 2006 as a physicist from Ghent University. From 2006 untill 2008 he studied engineering (option applied physics) at the Ghent University and received the Alcatel-Bell price for his master thesis: "Design and fabrication of a transceiver for Fiber-To-The-Home (FTTH) Optical Networks". Since 2008, he works in the Photonics Research Group of prof. Roel Baets at Ghent University under a specialization grant from the IWT (Institute for the promotion of innovation through science and technology in Flanders).
D. Van Thourhout received the degree in physical engineering and the Ph.D. degree from Ghent University, Ghent, Belgium in 1995 and 2000 respectively. From Oct. 2000 to Sep. 2002 he was with Lucent Technologies, Bell Laboratories, New Jersey, USA, working on the design, processing and characterization of $\mathrm{InP} / \mathrm{InGaAsP}$ monolithically integrated devices. In Oct. 2002 he joined the Department of Information Technology (INTEC), Ghent University, Belgium. Currently he is member of the permanent staff of the photonics group. He is lecturer or co-lecturer for 4 courses within the Ghent University Master in Photonics program (Microphotonics, Advanced Photonics Laboratory, Photonic Semiconductor Components and Technology). He is also coordinating the cleanroom activities of the research group. His research focuses on the design, fabrication and characterization of integrated photonic devices. Main topics involve Silicon nanophotonic devices and heterogeneous integration of InP-on-Silicon. Besides he is working on the development of new fabrication processes for photonic devices, e.g. based on focused ion beam etching and die-to-wafer bonding. $\mathrm{He}$ holds three patents, has authored and coauthored over 60 journal papers (see below) and has presented invited papers at several major conferences. He is member of IEEE LEOS and associate editor for IEEE Photonics Technology Letters. 